\title{
A mirror duality for families of $K 3$ surfaces associated to bimodular singularities
}

\author{
Makiko Mase \\ Key Words: K3 surfaces, toric varieties, Picard lattices \\ AMS MSC2010: 14J28 14M25 14C22
}

\begin{abstract}
Ebeling and Ploog 44 studied a duality of bimodular singularities which is part of the Berglund-Hübsch mirror symmetry. Mase and Ueda [7] showed that this duality leads to a polytope mirror symmetry of families of $K 3$ surfaces. We discuss in this article how this symmetry extends to a symmetry between lattices.
\end{abstract}

\section{Introduction}

Bimodular singularities classified by Arnold [1] have a duality studied by Ebeling and Ploog 4 analogous to Arnold's strange duality for unimodular singularities. Namely, a pair $\left((B, f),\left(B^{\prime}, f^{\prime}\right)\right)$ of singularities $B, B^{\prime}$ in $\mathbb{C}^{3}$ together with appropriate defining polynomials $f, f^{\prime}$ is dual if the matrices $A_{f}, A_{f^{\prime}}$ of exponents of $f$ and $f^{\prime}$ are transpose to each other. Moreover, in some cases, such polynomials are compactified as anticanonical members of 3-dimensional weighted projective spaces whose general members are Gorenstein $K 3$. The strange duality for unimodular singularities is related with the polytope mirror symmetry for families of $K 3$ surfaces that are obtained by compactifying the singularities by Kobayashi [6 in a certain sense. In the study of bimodular singularities, Mase and Ueda 7 extend the duality by Ebeling and Ploog to a polytope mirror symmetry of families of $K 3$ surfaces. More precisely, the following statement is shown :

Theorem [7] Let $\left((B, f),\left(B^{\prime}, f^{\prime}\right)\right)$ be a dual pair in the sense of [4] of singularities $B$ and $B^{\prime}$ together with their defining polynomials $f$ and $f^{\prime}$ that are respectively compactified into polynomials $F$ and $F^{\prime}$ as in [4]. Then, there exists a reflexive polytope $\Delta$ such that $\Delta_{F} \subset \Delta$ and $\Delta_{F^{\prime}} \subset \Delta^{*}$. Here, $\Delta_{F}$ and $\Delta_{F^{\prime}}$ are respectively the Newton polytopes of $F$ and of $F^{\prime}$, and $\Delta^{*}$ is the polar dual to $\Delta$.

In this article, we consider whether or not it is possible to extend the duality obtained in 7 further to the lattice mirror symmetry of families of $K 3$ surfaces. More precisely, our problem is stated as follows:

Problem Let $\Delta$ be a reflexive polytope as in [7]. Does there exist general members $S \in \mathcal{F}_{\Delta}$ and $S^{\prime} \in \mathcal{F}_{\Delta^{*}}$ such that an isometry $T(\widetilde{S}) \simeq \operatorname{Pic}\left(\widetilde{S^{\prime}}\right) \oplus U$ holds 
? Here, $\mathcal{F}_{\Delta}$ and $\mathcal{F}_{\Delta^{*}}$ are families of $K 3$ surfaces associated to the polytopes $\Delta$ and $\Delta^{*}, \widetilde{S}$ denotes the minimal model of $S$, Pic $(\widetilde{S})$ and $T(\widetilde{S})$ are respectively the Picard and transcendental lattice of $\widetilde{S}$.

The problem is answered in Theorem 3.2 together with an explicit description in Proposition 3.1 of the Picard lattices $\operatorname{Pic}(\Delta)$ and $\operatorname{Pic}\left(\Delta^{*}\right)$ defined in section 3, with ranks $\rho(\Delta)$ and $\rho\left(\Delta^{*}\right)$, of the minimal model of appropriate general members in the families. The main result of this article is summarized here. In the sequel, the names of singularities follow Arnold [1, and singularities in a same row of Table 1 are dual to each other in the sense of 4 .

Proposition 3.1 and Theorem 3.2 Let $\Delta$ be the reflexive polytope obtained in [77. For the following transpose-dual pairs, the polar duality extends to a lattice mirror symmetry between the families $\mathcal{F}_{\Delta}$ and $\mathcal{F}_{\Delta^{*}}$, where the Picard lattices are given in Table 1, Here we use the notation $C_{8}^{6}:=\left(\begin{array}{cc}-4 & 1 \\ 1 & -2\end{array}\right)$.

\begin{tabular}{cccccc} 
Singularity & $\operatorname{Pic}(\Delta)$ & $\rho(\Delta)$ & $\rho\left(\Delta^{*}\right)$ & $\operatorname{Pic}\left(\Delta^{*}\right)$ & Singularity \\
\hline \hline$Q_{12}$ & $U \oplus E_{6} \oplus E_{8}$ & 16 & 4 & $U \oplus A_{2}$ & $E_{18}$ \\
\hline$Z_{1,0}$ & $U \oplus E_{7} \oplus E_{8}$ & 17 & 3 & $U \oplus A_{1}$ & $E_{19}$ \\
\hline$E_{20}$ & $U \oplus E_{8}^{\oplus 2}$ & 18 & 2 & $U$ & $E_{20}$ \\
\hline$Q_{2,0}$ & $U \oplus A_{6} \oplus E_{8}$ & 16 & 4 & $U \oplus C_{8}^{6}$ & $Z_{17}$ \\
\hline$E_{25}$ & $U \oplus E_{7} \oplus E_{8}$ & 17 & 3 & $U \oplus A_{1}$ & $Z_{19}$ \\
\hline$Q_{18}$ & $U \oplus E_{6} \oplus E_{8}$ & 16 & 4 & $U \oplus A_{2}$ & $E_{30}$ \\
\hline
\end{tabular}

Table 1: Picard lattices for lattice mirror symmetric pair

Section 2 is to define the polytope- and lattice- mirror theories in subsection 2.3 and to define the transpose duality following [4 in subsection 2.4 based on a brief introduction to lattice theory in subsection 2.1 and of toric geometry in subsection 2.2. where several formulas and results are stated without proof.

The main theorem of this article is stated in section 3 following auxiliary results. The facts introduced in the previous section are used in their proof.

Denote by $\Delta_{B}$ the reflexive polytope obtained in 7 for a singularity $B$. As is seen in Table 1, there are isometric Picard lattices $\operatorname{Pic}\left(\Delta_{Q_{12}}\right) \simeq \operatorname{Pic}\left(\Delta_{Q_{18}}\right)$, and $\operatorname{Pic}\left(\Delta_{Z_{1,0}}\right) \simeq \operatorname{Pic}\left(\Delta_{E_{25}}\right)$. We consider and affirmatively answer in Proposition 4.1 the following question as an application in section 4

Problem Are the families $\mathcal{F}_{\Delta_{Q_{12}}}\left(\operatorname{resp} . \mathcal{F}_{\Delta_{Z_{1,0}}}\right)$ and $\mathcal{F}_{\Delta_{Q_{18}}}\left(\right.$ resp. $\left.\mathcal{F}_{\Delta_{E_{25}}}\right)$ essentially the same in the sense that general members in these families are birationally equivalent?

\section{Acknowledgement}

The author thanks to the referee for his helpful comments particularly about the proof of the key Proposition 3.1 in the original manuscript. 


\section{Preliminary}

We start with having a consensus as to Gorenstein $K 3$ and $K 3$ surfaces.

Definition 2.1 A compact complex connected 2-dimensional algebraic variety $S$ with at most $A D E$ singularities is called Gorenstein $K 3$ if (i) $K_{S} \sim 0$; and (ii) $H^{1}\left(S, \mathcal{O}_{S}\right)=0$. If a Gorenstein $K 3$ surface $S$ is nonsingular, $S$ is simply called a $K 3$ surface.

\subsection{Brief lattice theory}

A lattice is a non-degenerate finitely-generated free $\mathbb{Z}$-module with a symmetric bilinear form called an intersection pairing. The discriminant group of a lattice $L$ is defined by $A_{L}:=L^{*} / L$, which is finitely-generated and abelian, where $L^{*}:=$ $\operatorname{Hom}(L, \mathbb{Z})$ is dual to $L$. It is known that the order $\left|A_{L}\right|$ of the discriminant group is equal to the determinant of any intersection matrix of $L$. Let us recall a standard lattice theory by Nikulin [8]:

Corollary 2.1 (Corollary 1.13.5-(1) [8]) If an even lattice $L$ of signature $\left(t_{+}, t_{-}\right)$satisfies $t_{+} \geq 1, t_{-} \geq 1$, and $t_{+}+t_{-} \geq 3+$ length $A_{L}$, then, there exists a lattice $T$ such that $L \simeq U \oplus T$, where $U$ is the hyperbolic lattice of rank 2 .

In particular, if an even lattice $L$ is of $\operatorname{rk} L>12, t_{+} \geq 1$, and $t_{-} \geq 1$, then, there exists a lattice $T$ such that $L \simeq U \oplus T$.

Suppose $L$ is a sublattice of a lattice $L^{\prime}$ with inclusion $\iota: L \hookrightarrow L^{\prime}$. Denote by $L_{L^{\prime}}^{\perp}$ the orthogonal complement of $L$ in $L^{\prime}$. The embedding $\iota$ is called primitive, and $L$ called a primitive sublattice of $L^{\prime}$ if the finite abelian group $L^{\prime} / \iota(L)$ is torsion-free. In other words, if there is no overlattice that is an intermediate lattice between $L$ and $L^{\prime}$ of rank equal to the rank of $L$. Note that if a direct sum $L_{1} \oplus L_{2}$ of lattices is a sublattice of $L^{\prime}$, then $\left(L_{1} \oplus L_{2}\right) \frac{\perp}{L^{\prime}} \simeq\left(L_{1}\right)^{\frac{\perp}{L^{\prime}}} \oplus\left(L_{2}\right)_{L^{\prime}}$.

For a $K 3$ surface $S$, it is known that $H^{2}(S, \mathbb{Z})$ with the intersection pairing is isometric to the $K 3$ lattice $\Lambda_{K 3}$ that is even unimodular of rank 22 and signature $(3,19)$, being $U^{\oplus 3} \oplus E_{8}^{\oplus 2}$, where $E_{8}$ is the negative-definite even unimodular lattice of rank 8 . There is a standard exact sequence $0 \rightarrow H^{1}\left(S, \mathcal{O}_{S}^{*}\right) \stackrel{c_{1}}{\rightarrow}$ $H^{2}(S, \mathbb{Z}) \rightarrow 0$ of cohomologies so $H^{1}\left(S, \mathcal{O}_{S}^{*}\right)$ is inherited a lattice structure from $H^{2}(S, \mathbb{Z})$. Define the Picard lattice $\operatorname{Pic}(S)$ of a $K 3$ surface $S$ as the group $c_{1}\left(H^{1,1}(S)\right) \cap H^{2}(S, \mathbb{Z})$ with the lattice structure. The rank of $\operatorname{Pic}(S)$ is called the Picard number, denoted by $\rho(S)$. The Picard lattice is hyperbolic since a $K 3$ surface is complex and algebraic, and is known to be a primitive sublattice of $\Lambda_{K 3}$ under a marking $H^{2}(S, \mathbb{Z}) \stackrel{\sim}{\rightarrow} \Lambda_{K 3}$.

If an even hyperbolic lattice $L$ of $\operatorname{rank} \operatorname{rk} L \leq 20$ has $\left|A_{L}\right|$ being square-free, $L$ is a primitive sublattice of $\Lambda_{K 3}$. Indeed, if so, for any lattice $L \subset L^{\prime \prime} \subset$ $\Lambda_{K 3}$ the general relation $\left|A_{L}\right|=\left[L^{\prime \prime}: L\right]^{2}\left|A_{L^{\prime \prime}}\right|$ implies $\left[L^{\prime \prime}: L\right]=1$ thus $L^{\prime \prime} \simeq L$. Hence there is no overlattice of $L$. Moreover, by surjectivity of the period mapping [2], there exists a $K 3$ surface $S$ such that $\operatorname{Pic}(S) \simeq L$. Let $M \subset \Lambda_{K 3}$ be a hyperbolic sublattice. A $K 3$ surface is $M$-polarised 3 if there exists a marking $\phi$ such that all divisors in $\phi^{-1}\left(C_{M}^{p o l}\right)$ are ample, where $C_{M}^{\text {pol }}$ is the positive cone in $M_{\mathbb{R}}$ minus $\bigcup_{d \in \Delta_{M}} H_{d}, \Delta_{M}=\{d \in M \mid d . d=-2\}$, and $H_{d}=\left\{x \in \mathbb{P}\left(\Lambda_{K 3}\right) \mid x . d=0\right.$ for all $\left.d \in \Delta_{M}\right\}$.

Nishiyama 9] gives the orthogonal complements of primitive sublattices of type $A D E$ of $E_{8}$ in possible cases. 
Lemma 2.1 (Lemma $4.3[9]$ ) There exist primitive embeddings of lattices of type $A D E$ into $E_{8}$ with orthogonal complements given as follows. All the notation follows Bourbaki except $C_{8}^{6}:=\left(\begin{array}{cc}-4 & 1 \\ 1 & -2\end{array}\right)$.
$\left(A_{1}\right)_{E_{8}}^{\perp} \simeq E_{7}$
$\left(A_{2}\right)_{E_{8}}^{\perp} \simeq E_{6}$
$\left(A_{3}\right)_{E_{8}}^{\frac{1}{E_{8}} \simeq D_{5}}$
$\left(A_{5}\right)_{E_{8}}^{\perp} \simeq A_{1} \oplus A_{2}$
$\left(A_{6}\right)_{E_{8}}^{\perp_{8}} \simeq C_{8}^{6}$
$\left(A_{7}\right)_{E_{8}}^{\perp} \simeq(-8)$
$\left(D_{4}\right)_{E_{8}}^{\frac{\perp}{E_{8}}} \simeq D_{4}$
$\left(D_{5}\right)_{E_{8}}^{\perp} \simeq A_{3}$
$\left(E_{6}\right)_{E_{8}}^{\perp} \simeq A_{2}$
$\left(E_{7}\right)_{E_{8}}^{\perp} \simeq A_{1}$
$\left(A_{4}\right) \frac{\perp}{E_{8}} \simeq A_{4}$
$\left(D_{6}\right)^{\frac{1}{E_{8}}} \simeq A_{1}^{\oplus 2} \quad\left(D_{7}\right)^{\frac{1}{E_{8}}} \simeq(-4)$

\section{$2.2 \quad$ Brief toric geometry}

Here we summarize toric divisors and $\Delta$-regularity. Let $M$ be a rank-3 lattice with the standard basis $\left\{e_{1}, e_{2}, e_{3}\right\}, N$ be its dual, and $():, M \times N \rightarrow \mathbb{Z}$ be the natural pairing. From now on, a polytope means a 3 -dimensional convex hull of finitely-many points in $\mathbb{Z}^{3}$ embedded into $\mathbb{R}^{3}$, namely, integral, and the origin is the only lattice point in the interior of it.

Let $\mathbb{P}_{\Delta}$ be the toric variety defined by a polytope $\Delta$ in $M \otimes_{\mathbb{Z}} \mathbb{R}$, to which one can associate a fan $\Sigma_{\Delta}$ whose one-dimensional cones, called one-simplices, are generated by primitive lattice vectors each of whose end-point is an intersection point of $N$ and an edge of its polar dual $\Delta^{*}$ defined by

$$
\Delta^{*}:=\{y \in N \otimes \mathbb{R} \mid(x, y) \geq-1 \text { for all } x \in \Delta\} .
$$

Let $\widetilde{\mathbb{P}_{\Delta}}$ be the toric resolution of singularities in $\mathbb{P}_{\Delta}$. A toric divisor is a divisor admitting the torus action, identified with the closure of the torus-action orbit of a one-simplex. Let $\operatorname{Div}_{\mathbb{T}}\left(\widetilde{\mathbb{P}_{\Delta}}\right)$ be the set of all toric divisors $D_{i}=\overline{\operatorname{orb}\left(\mathbb{R}_{\geq 0} v_{i}\right)}, i=$ $1, \ldots, s$ on $\widetilde{\mathbb{P}_{\Delta}}$, where $v_{i}$ is a primitive lattice vector, then, $-K_{\widetilde{\mathbb{P}_{\Delta}}}=\sum_{i=1}^{s} D_{i}$. By a standard exact sequence and a commutative diagram [10]

$$
\begin{aligned}
0 \rightarrow M & \rightarrow \operatorname{Div}_{\mathbb{T}}\left(\widetilde{\mathbb{P}_{\Delta}}\right) \\
\downarrow & \rightarrow \operatorname{Pic}\left(\widetilde{\mathbb{P}_{\Delta}}\right) \rightarrow 0 \\
& \rightarrow \bigoplus_{i=1}^{s} \mathbb{Z} D_{i} \rightarrow A_{2}\left(\widetilde{\mathbb{P}_{\Delta}}\right) \rightarrow 0
\end{aligned}
$$

there is a system of linear equations among toric divisors

$$
\sum_{i=1}^{s}\left(e_{j}, v_{i}\right) D_{i}=0, \quad j=1,2,3 .
$$

Thus the solution set of the linear system is generated by $(s-3)$ elements corresponding to divisors which generate the Picard group $\operatorname{Pic}\left(\widetilde{\mathbb{P}_{\Delta}}\right)$ of $\widetilde{\mathbb{P}_{\Delta}}$.

Definition 2.2 A polytope is reflexive if its polar dual is also integral.

The importance of that we consider reflexive polytopes is by the following:

Theorem 2.1 c.f. [?] The following conditions are equivalent:

(1) A polytope $\Delta$ is reflexive.

(2) The toric 3-fold $\mathbb{P}_{\Delta}$ is Fano, in particular, general anticanonical members of $\mathbb{P}_{\Delta}$ are Gorenstein $K 3$. 
For a reflexive polytope $\Delta$, denote by $\mathcal{F}_{\Delta}$ the family of hypersurfaces parametrised by the complete anticanonical linear system of $\mathbb{P}_{\Delta}$. Note that general members in $\mathcal{F}_{\Delta}$ are Gorenstein $K 3$ due to the previous theorem so that they are birationally equivalent to $K 3$ surfaces by the existence of crepant resolution. Thus we call the family $\mathcal{F}_{\Delta}$ a family of $K 3$ surfaces.

We recall from $\S 3$ of $[?]$ the notion of $\Delta$-regularity.

Definition 2.3 Let $F$ be a Laurent polynomial defining a hypersurface $Z_{F}$, whose Newton polytope is a polytope $\Delta$. The hypersurface $Z_{F}$ is called $\Delta$ regular if for every face $\Gamma$ of $\Delta$, the corresponding affine stratum $Z_{F, \Gamma}$ of $Z_{F}$ is either empty or a smooth subvariety of codimension 1 in the torus $\mathbb{T}_{\Gamma}$ that is contained in the affine variety associated to $\Gamma$.

It is shown in [?] that $\Delta$-regularity is a general condition, and singularities of all $\Delta$-regular members are simultaneously resolved by a toric desingularization of $\mathbb{P}_{\Delta}$. From now on, suppose a polytope $\Delta$ is reflexive and $S$ is a $\Delta$-regular member whose minimal model $\widetilde{S}$ is obtained by a toric resolution.

Definition 2.4 For a restriction $r: \widetilde{\mathbb{P}_{\Delta}} \rightarrow \widetilde{S}$, let $r_{*}: H^{1,1}\left(\widetilde{\mathbb{P}_{\Delta}}\right) \rightarrow H^{1,1}(\widetilde{S})$ be the induced mapping. Define a lattice $L_{D}(\widetilde{S}):=r_{*}\left(H^{1,1}\left(\widetilde{\mathbb{P}_{\Delta}}\right)\right) \cap H^{2}(\widetilde{S}, \mathbb{Z})$ of the intersection of the image of $r_{*}$ and $H^{2}(\widetilde{S}, \mathbb{Z})$, and its orthogonal complement $L_{0}(\widetilde{S}):=L_{D}(\widetilde{S})_{H^{2}(\widetilde{S}, \mathbb{Z})}^{\perp}$ in $H^{2}(\widetilde{S}, \mathbb{Z})$.

It is known [6] that $\rho(\widetilde{S})$ and $\operatorname{rk} L_{0}(\widetilde{S})$ only depend on the number of lattice points in edges of $\Delta$ and $\Delta^{*}$. Thus we define the Picard number $\rho(\Delta):=\rho(\widetilde{S})$, and the rank rk $L_{0, \Delta}:=\operatorname{rk} L_{0}(\widetilde{S})$ associated to $\Delta$. More precisely, denote by $\Gamma^{*}$ in $\Delta^{*}$ the dual face to a face $\Gamma$ of $\Delta$, and $l^{*}(\Gamma)$ is the number of lattice points in the interior of $\Gamma$, and $\Delta^{[1]}$ the set of edges in $\Delta$. Let $s$ be the number of one-simplices of $\Sigma_{\Delta}$. Then

$$
\begin{aligned}
\operatorname{rk} L_{0, \Delta} & =\sum_{\Gamma \in \Delta^{[1]}} l^{*}(\Gamma) l^{*}\left(\Gamma^{*}\right)=\operatorname{rk} L_{0, \Delta^{*}}, \\
\rho(\Delta) & =s-3+\operatorname{rk} L_{0, \Delta} \\
\rho(\Delta)+\rho\left(\Delta^{*}\right) & =20+\operatorname{rk} L_{0, \Delta} .
\end{aligned}
$$

If $l^{*}\left(\Gamma^{*}\right)=n_{\Gamma}$ and $l^{*}(\Gamma)=m_{\Gamma}$ for an edge $\Gamma$ of $\Delta$, there is a singularity of type $A_{n_{\Gamma}+1}$ with multiplicity $m_{\Gamma}+1$ on an affine variety associated to $\Gamma$.

As we will see later, we only need formulas when $\operatorname{rk} L_{0, \Delta}=0$ for the intersection numbers of the divisors $\left\{r_{*} D_{i}\right\}$ in $H^{2}(\widetilde{S}, \mathbb{Z})$ given as follows.

$$
r_{*} D_{i}^{2}=r_{*} D_{i} \cdot r_{*} D_{i}= \begin{cases}2 l^{*}\left(v_{i}^{*}\right)-2 & \text { if } v_{i} \text { is a vertex of } \Delta^{*}, \\ -2 & \text { otherwise }\end{cases}
$$

If end-points of $v_{i}$ and $v_{j}$ are on the edge $\Gamma_{i j}^{*}$ of $\Delta^{*}$, then

$$
r_{*} D_{i} \cdot r_{*} D_{j}= \begin{cases}1 & \text { if } v_{i} \text { and } v_{j} \text { are next to each other, } \\ l^{*}\left(\Gamma_{i j}\right)+1 & \text { if } l^{*}\left(\Gamma_{i j}^{*}\right)=0, \text { and } v_{i}, v_{j} \text { are both vertices, }(6) \\ 0 & \text { otherwise. }\end{cases}
$$

The Picard lattices of the minimal models of any $\Delta$-regular members, which are generated by components of restricted toric divisors, are isometric. Define 
the Picard lattice $\operatorname{Pic}(\Delta)$ of the family $\mathcal{F}_{\Delta}$ as the Picard lattice of the minimal model of a $\Delta$-regular member with rank $\rho(\Delta)$. The orthogonal complement $T(\Delta)=\operatorname{Pic}(\Delta)_{\Lambda_{K 3}}^{\perp}$ is called the transcendental lattice of $\mathcal{F}_{\Delta}$.

\subsection{Mirrors}

We define the polytope- and lattice-mirror theories.

\subsubsection{Polytope Mirror}

We focus on polytopes that "represent" the anticanonical members in a toric variety as is seen in subsection 2.2

Definition 2.5 A pair $\left(\Delta_{1}, \Delta_{2}\right)$ of reflexive polytopes or a pair $\left(\mathcal{F}_{\Delta_{1}}, \mathcal{F}_{\Delta_{2}}\right)$ of families of $K 3$ surfaces associated to $\Delta_{1}$ and $\Delta_{2}$ is called polytope mirror symmetric if an isometry $\Delta_{1} \simeq \Delta_{2}^{*}$ holds.

\subsubsection{Lattice Mirror}

For a $K 3$ surface $S, \operatorname{Pic}(S)$ is the Picard lattice, and $T(S)=\operatorname{Pic}(S)_{\Lambda_{K 3}}^{\perp}$ is the transcendental lattice. A mirror for family of $M$-polarised $K 3$ surfaces is defined when $M$ is a sublattice of $\Lambda_{K 3}$ in general [3]. Here, we deal with the most strict case, namely, mirror for $K 3$ surfaces with Picard lattice as their polarisation.

Definition 2.6 (1) A pair $\left(S, S^{\prime}\right)$ of $K 3$ surfaces is called lattice mirror symmetric if an isometry $T(S) \simeq \operatorname{Pic}\left(S^{\prime}\right) \oplus U$ holds.

(2) A pair $\left(\mathcal{F}, \mathcal{F}^{\prime}\right)$ of families whose general members are Gorenstein $K 3$ surfaces is lattice mirror symmetric if there exist general members $S \in \mathcal{F}$ and $S^{\prime} \in \mathcal{F}^{\prime}$ pair of whose minimal models is lattice mirror symmetric.

Note that a lattice mirror pair $\left(S, S^{\prime}\right)$ of $K 3$ surfaces satisfies, by definition, $\operatorname{rk} \operatorname{Pic}\left(S^{\prime}\right)+2=\operatorname{rk} T(S)=22-\operatorname{rk} \operatorname{Pic}(S)$, thus

$$
\rho\left(S^{\prime}\right)+\rho(S)=20 .
$$

\subsection{Bimodular singularities and the transpose duality}

Being classified by Arnold [1 in 1970's, bimodular singularities have two specific classes: quadrilateral and exceptional. Quadrilateral bimodular singularities are 6 in number with exceptional divisor of type $I_{0}^{*}$, whilst exceptional are 14 in number with exceptional divisor of type $I I^{*}, I I I^{*}$ or $I V^{*}$ in Kodaira's notation.

A non-degenerate polynomial $f$ in three variables is called invertible if $f$ has three terms $f=\sum_{j=1}^{3} x^{a_{1 j}} y^{a_{2 j}} z^{a_{3 j}}$ such that its matrix $A_{f}:=\left(a_{i j}\right)_{1 \leq i, j \leq 3}$ of exponents is invertible in $G L_{3}(\mathbb{Q})$.

Definition 2.7 c.f. 4 Let $B=(0,(f=0))$ and $B^{\prime}=\left(0,\left(f^{\prime}=0\right)\right)$ be germs of singularities in $\mathbb{C}^{3}$. A pair $\left(B, B^{\prime}\right)$ of singularities is called transpose dual if the following three conditions are satisfied.

(1) Defining polynomials $f$ and $f^{\prime}$ are invertible.

(2) Matrices $A_{f}$ and $A_{f^{\prime}}$ of exponents of $f$ and $f^{\prime}$ are transpose to each other. 
(3) $f$ (resp. $f^{\prime}$ ) is compactified to a four-term polynomial $F$ (resp. $F^{\prime}$ ) in $\left|-K_{\mathbb{P}(a)}\right|$ (resp. $\left|-K_{\mathbb{P}(b)}\right|$ ), where $\mathbb{P}(a)$ (resp. $\left.\mathbb{P}(b)\right)$ is the 3-dimensional weighted projective space whose general members are Gorenstein $K 3$ with weight $a$ (resp. $b$ ) out of the list of 95 weights classified by [12] [5] [11.

Condition (1) and (2) is said that they are Berglund-Hübsch mirror symmetric. Ebeling-Ploog [4] show that there are 16 transpose-dual pairs among quadrilateral and exceptional bimodular singularities, exceptional unimodular singularities, and the singularities $E_{25}, E_{30}, X_{2,0}$, and $Z_{2,0}$.

\section{The transpose dual and the lattice mirror}

For a transpose-dual pair $\left(B, B^{\prime}\right)$ of defining polynomial $f$ (resp. $\left.f^{\prime}\right)$ being compactified to a polynomial $F$ (resp. $F^{\prime}$ ), consider the Newton polytope $\Delta_{F}$ (resp. $\Delta_{F^{\prime}}$ ) of $F$ (resp. $F^{\prime}$ ) all of whose corresponding monomials are fixed by an automorphism action on $(F=0)$ (resp. $\left.\left(F^{\prime}=0\right)\right)$.

Remark 1 A compactified member $F$ to $f$ does not always define a Gorenstein $K 3$ surface because $\Delta_{F}$ may not be reflexive.

However, the Newton polytopes are extended to be reflexive and dual.

Theorem 3.1 [7] For each transpose-dual pair $\left(B, B^{\prime}\right)$, there exists a reflexive polytope $\Delta$ such that $\Delta_{F} \subset \Delta$ and $\Delta_{F^{\prime}} \subset \Delta^{*}$.

Computing Picard lattices is generally difficult, but it seems possible for $\Delta$ regular members by subsection 2.2. Let us reformulate our problem.

Problem For a polytope $\Delta$ obtained in [7], is a pair $\left(\widetilde{S}, \widetilde{S}^{\prime}\right)$ of minimal models of $\Delta$-regular $S \in \mathcal{F}_{\Delta}$ and $\Delta^{*}$-regular $S^{\prime} \in \mathcal{F}_{\Delta^{*}}$ lattice mirror symmetric ?

First we study the rank rk $L_{0, \Delta}$.

Lemma 3.1 The list of $\operatorname{rk} L_{0, \Delta}$ for the reflexive polytope $\Delta$ obtained in [7] is given in Table Q.

\begin{tabular}{cccc} 
transpose-dual pair & rk $L_{0, \Delta}$ & transpose-dual pair & rk $L_{0, \Delta}$ \\
\hline \hline$\left(Q_{12}, E_{18}\right)$ & 0 & $\left(Z_{17}, Q_{2,0}\right)$ & 2 \\
\hline$\left(Z_{1,0}, E_{19}\right)$ & 0 & $\left(U_{1,0}, U_{1,0}\right)$ & 2 \\
\hline$\left(E_{20}, E_{20}\right)$ & 0 & $\left(U_{16}, U_{16}\right)$ & 2 \\
\hline$\left(Q_{2,0}, Z_{17}\right)$ & 0 & $\left(Q_{17}, Z_{2,0}\right)$ & 2 \\
\hline$\left(E_{25}, Z_{19}\right)$ & 0 & $\left(W_{1,0}, W_{1,0}\right)$ & 3 \\
\hline$\left(Q_{18}, E_{30}\right)$ & 0 & $\left(W_{17}, S_{1,0}\right)$ & 5 \\
\hline$\left(Z_{1,0}, Z_{1,0}\right)$ & 1 & $\left(W_{18}, W_{18}\right)$ & 6 \\
\hline$\left(Z_{13}, J_{3,0}\right)$ & 2 & $\left(S_{17}, X_{2,0}\right)$ & 6 \\
\hline
\end{tabular}

Table 2: $\operatorname{rk} L_{0, \Delta}$

ProOF. The assertion follows from direct and case-by-case computation by formula (2) in subsection 2.2 
1. $\left(Q_{12}, E_{18}\right)$ The polytope $\Delta$ is given in subsection 4.7 of 7 . There is no contribution to rk $L_{0, \Delta}$ since $l^{*}(\Gamma)$ or $l^{*}\left(\Gamma^{*}\right)$ is zero for any edge $\Gamma$. Thus, rk $L_{0, \Delta}=0$ by formula (2).

Similar for the cases $\left(Z_{1,0}, E_{19}\right),\left(E_{20}, E_{20}\right),\left(Q_{2,0}, Z_{17}\right),\left(E_{25}, Z_{19}\right),\left(Q_{18}, E_{30}\right)$. 2. $\left(Z_{13}, J_{3,0}\right)$ The polytope $\Delta$ is given in subsection 4.1 of [7]. The only contribution to rk $L_{0, \Delta}$ is by an edge $\Gamma$ between vertices $(0,0,1)$ and $(-2,-6,-9)$, whose dual $\Gamma^{*}$ is between $(8,-1,-1)$ and $(-1,2,-1)$ so $l^{*}(\Gamma)=1$ and $l^{*}\left(\Gamma^{*}\right)=$ 2. Thus, $r k L_{0, \Delta}=1 \times 2=2$ by formula (2).

Similar for the cases $\left(Z_{1,0}, Z_{1,0}\right),\left(Z_{17}, Q_{2,0}\right),\left(U_{1,0}, U_{1,0}\right),\left(U_{16}, U_{16}\right),\left(Q_{17}, Z_{2,0}\right)$, $\left(W_{1,0}, W_{1,0}\right),\left(W_{17}, S_{1,0}\right),\left(W_{18}, W_{18}\right),\left(S_{17}, X_{2,0}\right)$.

Corollary 3.1 No $\Delta$ - and $\Delta^{*}$-regular members for transpose-dual pairs $\left(Z_{13}, J_{3,0}\right)$, $\left(Z_{1,0}, Z_{1,0}\right),\left(Z_{17}, Q_{2,0}\right),\left(U_{1,0}, U_{1,0}\right),\left(U_{16}, U_{16}\right),\left(Q_{17}, Z_{2,0}\right),\left(W_{1,0}, W_{1,0}\right),\left(W_{17}, S_{1,0}\right)$, $\left(W_{18}, W_{18}\right),\left(S_{17}, X_{2,0}\right)$ admit a lattice mirror symmetry.

Proof. For each $\Delta$ associated to the presented pairs, by formula (4),

$$
\rho(\Delta)+\rho\left(\Delta^{*}\right)=20+\operatorname{rk} L_{0, \Delta}>20
$$

since rk $L_{0, \Delta}>0$ by Lemma 3.1. Thus, the equation (7) does not hold. Therefore, $\Delta$ - and $\Delta^{*}$-regular members do not admit a lattice mirror symmetry.

Corollary 3.2 The restriction mapping $r_{*}: \operatorname{Pic}\left(\widetilde{\mathbb{P}_{\Delta}}\right) \rightarrow \operatorname{Pic}(\widetilde{S})$, for $\Delta$-regular $S \in \mathcal{F}_{\Delta}$ is surjective for the transpose-dual pairs $\left(Q_{12}, E_{18}\right),\left(Z_{1,0}, E_{19}\right),\left(E_{20}, E_{20}\right)$, $\left(Q_{2,0}, Z_{17}\right),\left(E_{25}, Z_{19}\right),\left(Q_{18}, E_{30}\right)$.

Proof. By Lemma 3.1, rk $L_{0, \Delta}=0$ for each case. By definition, $\operatorname{rk} L_{0, \Delta}$ is equal to the rank of the orthogonal complement of $r_{*}\left(H^{1,1}\left(\widetilde{\mathbb{P}_{\Delta}}\right)\right)$ in $H^{2}(\widetilde{S}, \mathbb{Z})$. Thus, that $\operatorname{rk} L_{0, \Delta}=0$ means that $r_{*}$ is surjective.

By Corollary 3.1, we may only focus on the transpose-dual pairs appearing in Corollary 3.2, whose statement means moreover that $\operatorname{Pic}(\Delta)$ is generated by restricted toric divisors generating $\operatorname{Pic}\left(\widetilde{\mathbb{P}_{\Delta}}\right)$, and analogous to $\Delta^{*}$.

Let $A_{L}$ denote the discriminant group, $q_{L}$ the quadratic form, and $\operatorname{discr} L$ the discriminant of a lattice $L$. If $p=\operatorname{discr} L$ is prime, then $A_{L} \simeq \mathbb{Z} / p \mathbb{Z}$. Before stating our main results, note a fact in Proposition 1.6.1 in [8]. Suppose that lattices $S$ and $T$ are primitively embedded into the $K 3$ lattice $\Lambda_{K 3}$. If $A_{S} \simeq A_{T}$ and $q_{S}=-q_{T}$, then, it is determined that the orthogonal complement $S_{\Lambda_{K 3}}^{\perp}$ in $\Lambda_{K 3}$ is $T$. And $q_{S}=-q_{T}$ if and only if $\operatorname{discr} S=-\operatorname{discr} T$.

Proposition 3.1 The Picard lattice $\operatorname{Pic}\left(\Delta^{*}\right)$ for $\Delta$ in Corollary 3.2 is as in Table 3, where singularities in a row are transpose-dual. In each case, one gets

$$
\operatorname{discr} \operatorname{Pic}(\Delta)=-\operatorname{discr}\left(U \oplus \operatorname{Pic}\left(\Delta^{*}\right)\right), \text { and } A_{\operatorname{Pic}(\Delta)} \simeq A_{U \oplus \operatorname{Pic}\left(\Delta^{*}\right)} .
$$


Denote $C_{8}^{6}:=\left(\begin{array}{cc}-4 & 1 \\ 1 & -2\end{array}\right)$.

\begin{tabular}{cccc} 
Singularity & $\rho\left(\Delta^{*}\right)$ & $\operatorname{Pic}\left(\Delta^{*}\right)$ & Singularity \\
\hline \hline$Q_{12}$ & 4 & $U \oplus A_{2}$ & $E_{18}$ \\
\hline$Z_{1,0}$ & 3 & $U \oplus A_{1}$ & $E_{19}$ \\
\hline$E_{20}$ & 2 & $U$ & $E_{20}$ \\
\hline$Q_{2,0}$ & 4 & $U \oplus C_{8}^{6}$ & $Z_{17}$ \\
\hline$E_{25}$ & 3 & $U \oplus A_{1}$ & $Z_{19}$ \\
\hline$Q_{18}$ & 4 & $U \oplus A_{2}$ & $E_{30}$ \\
\hline
\end{tabular}

Table 3: $\operatorname{Pic}\left(\Delta^{*}\right)$ for $\Delta$ in Corollary 3.2

Proof.

1. $Q_{12}$ and $E_{18}$ The polytope $\Delta$ is given in subsection 4.7 of $[7$ and the associated toric 3 -fold has 19 toric divisors $D_{i}$ corresponding to the one-simplices generated by vectors

$$
\begin{array}{lll}
m_{1}=(1,2,2) & m_{2}=(0,1,1) & m_{3}=(-8,-11,-9) \\
m_{4}=(1,-2,0) & m_{5}=(1,1,0) & m_{6}=(-4,-5,-4) \\
m_{7}=(-7,-10,-8) & m_{8}=(-6,-9,-7) & m_{9}=(-5,-8,-6) \\
m_{10}=(-4,-7,-5) & m_{11}=(-3,-6,-4) & m_{12}=(-2,-5,-3) \\
m_{13}=(-1,-4,-2) & m_{14}=(0,-3,-1) & m_{15}=(1,0,1) \\
m_{16}=(-5,-7,-6) & m_{17}=(-2,-3,-3) & m_{18}=(1,-1,0) \\
m_{19}=(1,0,0) & &
\end{array}
$$

and by solving the linear system (10) : $\sum_{i=1}^{19}\left(e_{j}, m_{i}\right) D_{i}=0 \quad(j=1,2,3)$, we get linear relations among toric divisors

$$
\begin{aligned}
& D_{1} \sim-9 D_{4}+3 D_{5}-D_{7}-2 D_{8}-3 D_{9}-4 D_{10}-5 D_{11}-6 D_{12}-7 D_{13}-8 D_{14} \\
& -5 D_{15}+D_{16}+2 D_{17}-5 D_{18}-D_{19} \\
& D_{2} \sim D_{3}+10 D_{4}-2 D_{5}+2 D_{7}+3 D_{8}+4 D_{9}+5 D_{10}+6 D_{11}+7 D_{12}+8 D_{13} \\
& +9 D_{14}+5 D_{15}-D_{17}+6 D_{18}+2 D_{19}, \\
& D_{6} \sim-2 D_{3}-2 D_{4}+D_{5}-2 D_{7}-2 D_{8}-2 D_{9}-2 D_{10}-2 D_{11}-2 D_{12}-2 D_{13} \\
& -2 D_{14}-D_{15}-D_{16}-D_{18} \text {. }
\end{aligned}
$$

So the set $\left\{D_{i} \mid i \neq 1,2,6\right\}$ of toric divisors is linearly independent. Let $L$ be the lattice generated by the set $\left\{r_{*} D_{i} \mid i \neq 1,2,6\right\}$ of their restrictions to a $\Delta$ regular member. We shall check that $L$ is primitively embedded into the $K 3$ lattice to show that $L$ is indeed the Picard lattice of the family $\mathcal{F}_{\Delta}$. By computer calculation with formulas (5) and (6), the determinant of an intersection matrix 
of $L$ is -3 since this matrix is given by

$$
\left(\begin{array}{cccccccccccccccc}
-2 & 0 & 0 & 1 & 0 & 0 & 0 & 0 & 0 & 0 & 0 & 0 & 1 & 0 & 0 & 0 \\
0 & -2 & 0 & 0 & 0 & 0 & 0 & 0 & 0 & 0 & 1 & 1 & 0 & 0 & 1 & 0 \\
0 & 0 & 0 & 0 & 0 & 0 & 0 & 0 & 0 & 0 & 0 & 0 & 0 & 1 & 0 & 1 \\
1 & 0 & 0 & -2 & 1 & 0 & 0 & 0 & 0 & 0 & 0 & 0 & 0 & 0 & 0 & 0 \\
0 & 0 & 0 & 1 & -2 & 1 & 0 & 0 & 0 & 0 & 0 & 0 & 0 & 0 & 0 & 0 \\
0 & 0 & 0 & 0 & 1 & -2 & 1 & 0 & 0 & 0 & 0 & 0 & 0 & 0 & 0 & 0 \\
0 & 0 & 0 & 0 & 0 & 1 & -2 & 1 & 0 & 0 & 0 & 0 & 0 & 0 & 0 & 0 \\
0 & 0 & 0 & 0 & 0 & 0 & 1 & -2 & 1 & 0 & 0 & 0 & 0 & 0 & 0 & 0 \\
0 & 0 & 0 & 0 & 0 & 0 & 0 & 1 & -2 & 1 & 0 & 0 & 0 & 0 & 0 & 0 \\
0 & 0 & 0 & 0 & 0 & 0 & 0 & 0 & 1 & -2 & 1 & 0 & 0 & 0 & 0 & 0 \\
0 & 1 & 0 & 0 & 0 & 0 & 0 & 0 & 0 & 1 & -2 & 0 & 0 & 0 & 0 & 0 \\
0 & 1 & 0 & 0 & 0 & 0 & 0 & 0 & 0 & 0 & 0 & -2 & 0 & 0 & 0 & 0 \\
1 & 0 & 0 & 0 & 0 & 0 & 0 & 0 & 0 & 0 & 0 & 0 & -2 & 1 & 0 & 0 \\
0 & 0 & 1 & 0 & 0 & 0 & 0 & 0 & 0 & 0 & 0 & 0 & 1 & -2 & 0 & 0 \\
0 & 1 & 0 & 0 & 0 & 0 & 0 & 0 & 0 & 0 & 0 & 0 & 0 & 0 & -2 & 1 \\
0 & 0 & 1 & 0 & 0 & 0 & 0 & 0 & 0 & 0 & 0 & 0 & 0 & 0 & 1 & -2
\end{array}\right)
$$

Since the discriminant of $L$ is -3 that is square-free, there exists no overlattice of $L$; indeed, if $H \subset \Lambda_{K 3}$ were an overlattice of $L$, then, by the standard relation $-3=[H: L]^{2}$ discr $H$, we get $[H: L]=1$ and $\operatorname{discr} H=-3$ so that $L \simeq H$. Hence, $L$ is primitively embedded into the $K 3$ lattice. By construction, $L$ is indeed the Picard lattice of the family $\mathcal{F}_{\Delta}$.

The dual polytope $\Delta^{*}$ associates a toric 3 -fold with 7 toric divisors $D_{i}^{\prime}$ corresponding to the one-simplices generated by vectors

$$
\begin{array}{lll}
v_{1}=(1,1,-2) & v_{2}=(1,-2,1) & v_{3}=(2,-3,2) \\
v_{5}=(-1,0,0) & v_{6}=(1,0,-1) & v_{7}=(1,-1,0)
\end{array}
$$

and by solving the linear system (10): $\sum_{i=1}^{7}\left(e_{j}, m_{i}\right) D_{i}^{\prime}=0 \quad(j=1,2,3)$, we get linear relations among toric divisors

$$
D_{1}^{\prime} \sim 2 D_{2}^{\prime}+3 D_{3}^{\prime}+D_{7}^{\prime}, \quad D_{4}^{\prime} \sim 3 D_{2}^{\prime}+4 D_{3}^{\prime}+D_{6}^{\prime}+2 D_{7}^{\prime}, \quad D_{5}^{\prime} \sim D_{3}^{\prime} .
$$

Thus the set $\left\{D_{i}^{\prime} \mid i \neq 1,4,5\right\}$ of toric divisors is linearly independent. Let $L^{\prime}$ be the lattice generated by the set $\left\{r_{*} D_{i}^{\prime} \mid i \neq 1,4,5\right\}$ of their restrictions to a $\Delta^{*}$-regular member. By formulas (5) and (마), an intersection matrix associated to $L^{\prime}$ is given by

$$
\left(\begin{array}{cccc}
-2 & 1 & 0 & 1 \\
1 & 0 & 0 & 0 \\
0 & 0 & -2 & 1 \\
1 & 0 & 1 & -2
\end{array}\right)
$$

that is equivalent to $U \oplus A_{2}$ by re-taking the generators as

$$
\left\{r_{*} D_{2}^{\prime}+r_{*} D_{3}^{\prime}, r_{*} D_{3}^{\prime}, r_{*} D_{6}^{\prime}, r_{*} D_{3}^{\prime}+r_{*} D_{7}^{\prime}\right\} \text {. }
$$

Since the discriminant of $L^{\prime}$ is -3 that is square-free, there exists no overlattice of $L^{\prime}$; indeed, if $H^{\prime} \subset \Lambda_{K 3}$ were an overlattice of $L^{\prime}$, then, by the standard relation $-3=\left[H^{\prime}: L^{\prime}\right]^{2} \operatorname{discr} H^{\prime}$, we get $\left[H^{\prime}: L^{\prime}\right]=1$ and $\operatorname{discr} H^{\prime}=-3$ so that $H^{\prime} \simeq L^{\prime}$. Hence, $L^{\prime}$ is primitively embedded into the $K 3$ lattice. By 
construction, $L^{\prime}$ is indeed the Picard lattice of the family $\mathcal{F}_{\Delta^{*}}$. Therefore $\operatorname{Pic}\left(\Delta^{*}\right)=L^{\prime}=\left(\mathbb{Z}^{4}, U \oplus A_{2}\right)$.

Similarly, the lattice $U \oplus \operatorname{Pic}\left(\Delta^{*}\right)$ is also primitively embedded into the $K 3$ lattice since it is of signature $(2,4)$ and is of discriminant 3 . Besides, $\operatorname{discr}(U \oplus$ $\left.\operatorname{Pic}\left(\Delta^{*}\right)\right)=3=-\operatorname{discr} \operatorname{Pic}(\Delta)$, and moreover, $A_{\operatorname{Pic}(\Delta)} \simeq A_{U \oplus \operatorname{Pic}\left(\Delta^{*}\right)} \simeq \mathbb{Z} / 3 \mathbb{Z}$.

2. $Z_{1,0}$ and $E_{19}$ The polytope $\Delta$ is given in subsection 4.8 of [7] and the associated toric 3 -fold has 20 toric divisors $D_{i}$ corresponding to

$$
\begin{array}{lll}
m_{1}=(1,-1,2) & m_{2}=(0,-1,1) & m_{3}=(0,0,1) \\
m_{4}=(4,2,-1) & m_{5}=(-6,2,-11) & m_{6}=(2,0,1) \\
m_{7}=(3,1,0) & m_{8}=(-2,0,-3) & m_{9}=(-4,1,-7) \\
m_{10}=(2,1,0) & m_{11}=(-3,1,-5) & m_{12}=(3,2,-2) \\
m_{13}=(2,2,-3) & m_{14}=(1,2,-4) & m_{15}=(0,2,-5) \\
m_{16}=(-1,2,-6) & m_{17}=(-2,2,-7) & m_{18}=(-3,2,-8) \\
m_{19}=(-4,2,-9) & m_{20}=(-5,2,-10) &
\end{array}
$$

and $\left\{D_{i} \mid i \neq 1,2,3\right\}$ is linearly independent by system (1). The lattice $L:=$ $\left\langle r_{*} D_{i} \mid i \neq 1,2,3\right\rangle_{\mathbb{Z}}$ has $\operatorname{rk} L=17$, and $\operatorname{discr} L=2$ by an explicit calculation of its intersection matrix using formulas (5) and (6). Since the discriminant of $L$ is square-free, $L$ is primitively embedded into the $K 3$ lattice, and thus $L=\operatorname{Pic}(\Delta)$.

The dual polytope $\Delta^{*}$ associates a toric 3 -fold with 6 toric divisors $D_{i}^{\prime}$ corresponding to

$$
\begin{array}{lll}
v_{1}=(1,-3,-1) & v_{2}=(2,0,-1) & v_{3}=(1,0,-1) \\
v_{4}=(0,-1,-1) & v_{5}=(-1,2,1) & v_{6}=(0,1,0)
\end{array}
$$

and $\left\{D_{i}^{\prime} \mid i \neq 1,2,5\right\}$ is linearly independent by system (11). By re-taking the generators as $\left\{r_{*} D_{3}^{\prime}+r_{*} D_{4}^{\prime}, r_{*} D_{4}^{\prime}, r_{*} D_{6}^{\prime}-r_{*} D_{4}^{\prime}\right\}$, the lattice $L^{\prime}:=\left\langle r_{*} D_{i}^{\prime}\right| i \neq$ $1,2,5\rangle_{\mathbb{Z}}$ has an intersection matrix $U \oplus A_{1}$. Since $\operatorname{rk} L^{\prime}=3$, and discr $L^{\prime}=2$, which is square-free, $L^{\prime}$ is primitively embedded into the $K 3$ lattice, and thus $\operatorname{Pic}\left(\Delta^{*}\right)=L^{\prime}=\left(\mathbb{Z}^{3}, U \oplus A_{1}\right)$.

Similarly $U \oplus \operatorname{Pic}\left(\Delta^{*}\right)$ is primitively embedded into the $K 3$ lattice. Besides, $\operatorname{discr}\left(U \oplus \operatorname{Pic}\left(\Delta^{*}\right)\right)=-2=-\operatorname{discr} \operatorname{Pic}(\Delta)$, and moreover, $A_{\operatorname{Pic}(\Delta)} \simeq$ $A_{U \oplus \operatorname{Pic}\left(\Delta^{*}\right)} \simeq \mathbb{Z} / 2 \mathbb{Z}$.

3. $E_{20}$ and $E_{20}$ The polytope $\Delta$ is given in subsection 4.9 of [7] and the associated toric 3 -fold has 21 toric divisors $D_{i}$ corresponding to

$$
\begin{array}{lll}
m_{1}=(-1,-1,2) & m_{2}=(-1,-1,-1) & m_{3}=(-1,11,2) \\
m_{4}=(1,-1,0) & m_{5}=(-1,-1,1) & m_{6}=(-1,-1,0) \\
m_{7}=(-1,3,0) & m_{8}=(-1,7,1) & m_{9}=(0,-1,1) \\
m_{10}=(0,5,1) & m_{11}=(-1,0,2) & m_{12}=(-1,1,2) \\
m_{13}=(-1,2,2) & m_{14}=(-1,3,2) & m_{15}=(-1,4,2) \\
m_{16}=(-1,5,2) & m_{17}=(-1,6,2) & m_{18}=(-1,7,2) \\
m_{19}=(-1,8,2) & m_{20}=(-1,9,2) & m_{21}=(-1,10,2)
\end{array}
$$

and $\left\{D_{i} \mid i \neq 10,13,14\right\}$ is linearly independent by system (11). The lattice $L:=$ $\left\langle r_{*} D_{i} \mid i \neq 10,13,14\right\rangle_{\mathbb{Z}}$ has $\operatorname{rk} L=18$, and $\operatorname{discr} L=-1$ by an explicit calculation of its intersection matrix using formulas (5) and (6). As it being unimodular, $L$ is primitively embedded into the $K 3$ lattice, and thus $L=\operatorname{Pic}(\Delta)$. 
The dual polytope $\Delta^{*}$ associates a toric 3 -fold with 5 toric divisors $D_{i}^{\prime}$ corresponding to

$$
\begin{array}{ll}
v_{1}=(0,1,0) & v_{2}=(1,0,0) \\
v_{4}=(-2,-1,4) & v_{5}=(-1,0,2)
\end{array} \quad v_{3}=(-1,0,-1)
$$

and $\left\{D_{i}^{\prime} \mid i \neq 1,2,3\right\}$ is linearly independent by system (1). By an intersection matrix computed by formulas (5) and (6), the lattice $L^{\prime}:=\left\langle r_{*} D_{i}^{\prime} \mid i \neq 1,2,3\right\rangle_{\mathbb{Z}}$ has $\operatorname{discr} L^{\prime}=-1, \operatorname{rk} L^{\prime}=2$, and $L^{\prime}$ is even. By the classification of even unimodular lattices, $L^{\prime}$ is isometric to $U$, which is primitively embedded into the $K 3$ lattice, and thus $\operatorname{Pic}\left(\Delta^{*}\right)=L^{\prime}=\left(\mathbb{Z}^{2}, U\right)$.

Similarly, $U \oplus \operatorname{Pic}\left(\Delta^{*}\right)$ is primitively embedded into the $K 3$ lattice. Besides, $\operatorname{discr}\left(U \oplus \operatorname{Pic}\left(\Delta^{*}\right)\right)=1=-\operatorname{discr} \operatorname{Pic}(\Delta)$, and moreover, $A_{\operatorname{Pic}(\Delta)} \simeq$ $A_{U \oplus \operatorname{Pic}\left(\Delta^{*}\right)} \simeq\{0\}$.

4. $Q_{2,0}$ and $Z_{17}$ The polytope $\Delta$ is given in subsection 4.10 of [7] and the associated toric 3 -fold has 19 toric divisors $D_{i}$ corresponding to

$$
\begin{array}{lll}
m_{1}=(0,1,1) & m_{2}=(1,2,2) & m_{3}=(1,1,2) \\
m_{4}=(0,-1,0) & m_{5}=(-6,-7,-9) & m_{6}=(1,0,-2) \\
m_{7}=(1,0,1) & m_{8}=(1,1,0) & m_{9}=(-2,-3,-3) \\
m_{10}=(-4,-5,-6) & m_{11}=(1,0,-1) & m_{12}=(1,0,0) \\
m_{13}=(-5,-6,-8) & m_{14}=(-4,-5,-7) & m_{15}=(-3,-4,-6) \\
m_{16}=(-2,-3,-5) & m_{17}=(-1,-2,-4) & m_{18}=(0,-1,-3) \\
m_{19}=(-3,-3,-4) & &
\end{array}
$$

and $\left\{D_{i} \mid i \neq 1,2,3\right\}$ is linearly independent by system (1). The lattice $L:=$ $\left\langle r_{*} D_{i} \mid i \neq 1,2,3\right\rangle_{\mathbb{Z}}$ has $\operatorname{rk} L=16$, and $\operatorname{discr} L=-7$ by an explicit calculation of its intersection matrix using formulas (5) and (6). Since the discriminant of $L$ is square-free, $L$ is primitively embedded into the $K 3$ lattice, and thus $L=\operatorname{Pic}(\Delta)$

The dual polytope $\Delta^{*}$ associates a toric 3 -fold with 7 toric divisors $D_{i}^{\prime}$ corresponding to

$$
\begin{array}{lll}
v_{1}=(1,-2,1) & v_{2}=(-1,1,0) & v_{3}=(2,1,-2) \\
v_{5}=(0,1,-1) & v_{6}=(-1,0,0) & v_{7}=(1,-1,0)
\end{array}
$$

and $\left\{D_{i}^{\prime} \mid i \neq 1,2,6\right\}$ is linearly independent by system (1). By re-taking the generators as $\left\{r_{*} D_{3}^{\prime}, r_{*} D_{3}^{\prime}+r_{*} D_{4}^{\prime}, 2 r_{*} D_{3}^{\prime}+r_{*} D_{4}^{\prime}-r_{*} D_{5}^{\prime}, r_{*} D_{7}^{\prime}-r_{*} D_{3}^{\prime}\right\}$, the lattice $L^{\prime}:=\left\langle r_{*} D_{i}^{\prime} \mid i \neq 1,2,6\right\rangle$ has an intersection matrix $U \oplus C_{8}^{6}$. Since discr $L^{\prime}=-7$ is square-free, $L^{\prime}$ is primitively embedded into the $K 3$ lattice, and thus $\operatorname{Pic}\left(\Delta^{*}\right)=L^{\prime}=\left(\mathbb{Z}^{4}, U \oplus C_{8}^{6}\right)$.

Similarly $U \oplus \operatorname{Pic}\left(\Delta^{*}\right)$ is primitively embedded into the $K 3$ lattice. Besides, $\operatorname{discr}\left(U \oplus \operatorname{Pic}\left(\Delta^{*}\right)\right)=7=-\operatorname{discr} \operatorname{Pic}(\Delta)$, and moreover, $A_{\operatorname{Pic}(\Delta)} \simeq$ $A_{U \oplus \operatorname{Pic}\left(\Delta^{*}\right)} \simeq \mathbb{Z} / 7 \mathbb{Z}$. 
5. $E_{25}$ and $Z_{19}$ The polytope $\Delta$ is given in subsection 4.11 of 7 and the associated toric 3 -fold has 20 toric divisors $D_{i}$ corresponding to

$$
\begin{array}{lll}
m_{1}=(-1,2,0) & m_{2}=(-1,-1,9) & m_{3}=(-1,-1,-1) \\
m_{4}=(-1,2,-1) & m_{5}=(1,-1,-1) & m_{6}=(-1,1,3) \\
m_{7}=(-1,0,6) & m_{8}=(-1,-1,8) & m_{9}=(-1,-1,7) \\
m_{10}=(-1,-1,6) & m_{11}=(-1,-1,5) & m_{12}=(-1,-1,4) \\
m_{13}=(-1,-1,3) & m_{14}=(-1,-1,2) & m_{15}=(-1,-1,1) \\
m_{16}=(-1,-1,0) & m_{17}=(-1,0,-1) & m_{18}=(-1,1,-1) \\
m_{19}=(0,-1,4) & m_{20}=(0,-1,-1) &
\end{array}
$$

and $\left\{D_{i} \mid i \neq 1,4,5\right\}$ is linearly independent by system (1). The lattice $L:=$ $\left\langle r_{*} D_{i} \mid i \neq 1,4,5\right\rangle_{\mathbb{Z}}$ has $\operatorname{rk} L=17$, and $\operatorname{discr} L=2$ by an explicit calculation of its intersection matrix using formulas (5) and (6). Since the discriminant of $L$ is square-free, $L$ is primitively embedded into the $K 3$ lattice, and thus $L=\operatorname{Pic}(\Delta)$.

The dual polytope $\Delta^{*}$ associates a toric 3 -fold with 6 toric divisors $D_{i}^{\prime}$ corresponding to

$$
\begin{array}{lll}
v_{1}=(0,1,0) & v_{2}=(0,0,1) & v_{3}=(-3,-2,0) \\
v_{4}=(-5,-3,-1) & v_{5}=(1,0,0) & v_{6}=(-1,-1,0)
\end{array}
$$

and $\left\{D_{i}^{\prime} \mid i \neq 1,2,3\right\}$ is linearly independent by system (1). By re-taking the generators as $\left\{r_{*} D_{4}^{\prime}, r_{*} D_{5}^{\prime}-8 r_{*} D_{4}^{\prime}, r_{*} D_{6}^{\prime}-r_{*} D_{4}^{\prime}\right\}$, the lattice $L^{\prime}:=\left\langle r_{*} D_{i}^{\prime}\right| i \neq$ $1,2,3\rangle_{\mathbb{Z}}$ has an intersection matrix $U \oplus A_{1}$. Since discr $L^{\prime}=2$ is square-free, $L^{\prime}$ is primitively embedded into the $K 3$ lattice, and thus $\operatorname{Pic}\left(\Delta^{*}\right)=L^{\prime}=$ $\left(\mathbb{Z}^{3}, U \oplus A_{1}\right)$.

Similarly, $U \oplus \operatorname{Pic}\left(\Delta^{*}\right)$ is primitively embedded into the $K 3$ lattice. Besides, $\operatorname{discr}\left(U \oplus \operatorname{Pic}\left(\Delta^{*}\right)\right)=-2=-\operatorname{discr} \operatorname{Pic}(\Delta)$, and moreover, $A_{\operatorname{Pic}(\Delta)} \simeq$ $A_{U \oplus \operatorname{Pic}\left(\Delta^{*}\right)} \simeq \mathbb{Z} / 2 \mathbb{Z}$.

6. $Q_{18}$ and $E_{30}$ The polytope $\Delta$ is given in subsection 4.13 of [7] and the associated toric 3 -fold has 19 toric divisors $D_{i}$ corresponding to

$$
\begin{array}{lll}
m_{1}=(1,-1,-1) & m_{2}=(-1,-1,-1) & m_{3}=(-1,-1,8) \\
m_{4}=(1,-1,0) & m_{5}=(-1,2,-1) & m_{6}=(0,-1,-1) \\
m_{7}=(-1,-1,0) & m_{8}=(-1,-1,1) & m_{9}=(-1,-1,2) \\
m_{10}=(-1,-1,3) & m_{11}=(-1,-1,4) & m_{12}=(-1,-1,5) \\
m_{13}=(-1,-1,6) & m_{14}=(-1,-1,7) & m_{15}=(0,-1,4) \\
m_{16}=(-1,0,-1) & m_{17}=(-1,1,-1) & m_{18}=(-1,0,5) \\
m_{19}=(-1,0,2) & &
\end{array}
$$

and $\left\{D_{i} \mid i \neq 1,4,5\right\}$ is linearly independent by system (11). The lattice $L:=$ $\left\langle r_{*} D_{i} \mid i \neq 1,4,5\right\rangle_{\mathbb{Z}}$ has $\operatorname{rk} L=16$, and $\operatorname{discr} L=-3$ by an explicit calculation of its intersection matrix using formulas (5) and (6). Since the discriminant of $L$ is square-free, $L$ is primitively embedded into the $K 3$ lattice, and thus $L=\operatorname{Pic}(\Delta)$.

The dual polytope $\Delta^{*}$ associates a toric 3 -fold with 7 toric divisors $D_{i}^{\prime}$ corresponding to

$$
\begin{aligned}
& v_{1}=(0,0,1) \quad v_{2}=(1,0,0) \quad v_{3}=(-4,-3,-1) \quad v_{4}=(-3,-2,0) \\
& v_{5}=(0,1,0) \quad v_{6}=(-2,-1,0) \quad v_{7}=(-1,0,0)
\end{aligned}
$$


and $\left\{D_{i}^{\prime} \mid i \neq 1,2,5\right\}$ is linearly independent by system (11). By re-taking the generators as $\left\{r_{*} D_{3}^{\prime}, r_{*} D_{4}^{\prime}+r_{*} D_{3}^{\prime}, r_{*} D_{6}^{\prime}-r_{*} D_{3}^{\prime}, r_{*} D_{7}^{\prime}\right\}$, the lattice $L^{\prime}:=$ $\left\langle r_{*} D_{i}^{\prime} \mid i \neq 1,2,5\right\rangle_{\mathbb{Z}}$ has an intersection matrix $U \oplus A_{2}$. Since $\operatorname{discr} L^{\prime}=-3$ is square-free, $L^{\prime}$ is primitively embedded into the $K 3$ lattice, and thus $\operatorname{Pic}\left(\Delta^{*}\right)=$ $L^{\prime}=\left(\mathbb{Z}^{4}, U \oplus A_{2}\right)$.

Similarly, $U \oplus \operatorname{Pic}\left(\Delta^{*}\right)$ is primitively embedded into the $K 3$ lattice. Besides, $\operatorname{discr}\left(U \oplus \operatorname{Pic}\left(\Delta^{*}\right)\right)=3=-\operatorname{discr} \operatorname{Pic}(\Delta)$, and moreover, $A_{\operatorname{Pic}(\Delta)} \simeq$ $A_{U \oplus \operatorname{Pic}\left(\Delta^{*}\right)} \simeq \mathbb{Z} / 3 \mathbb{Z}$.

Remark 2 The choice of $\Delta$ is not actually unique. However, for any possible reflexive polytopes $\Delta$ for transpose-dual pairs outside Table 3 , we have a relation $\rho(\Delta)+\rho\left(\Delta^{*}\right) \neq 20$.

Theorem 3.2 The families $\mathcal{F}_{\Delta}$ and $\mathcal{F}_{\Delta^{*}}$ are lattice mirror symmetric for polytopes $\Delta$ in Corollary [3.2, that is, $\operatorname{Pic}(\Delta) \simeq\left(U \oplus \operatorname{Pic}\left(\Delta^{*}\right)\right)_{\Lambda_{K 3}}^{\perp}$. Explicitely, the lattices $\operatorname{Pic}(\Delta)$ are given as in the Table 4

\begin{tabular}{cccccc} 
Singularity & $\operatorname{Pic}(\Delta)$ & $\rho(\Delta)$ & $\rho\left(\Delta^{*}\right)$ & $\operatorname{Pic}\left(\Delta^{*}\right)$ & Singularity \\
\hline \hline$Q_{12}$ & $U \oplus E_{6} \oplus E_{8}$ & 16 & 4 & $U \oplus A_{2}$ & $E_{18}$ \\
\hline$Z_{1,0}$ & $U \oplus E_{7} \oplus E_{8}$ & 17 & 3 & $U \oplus A_{1}$ & $E_{19}$ \\
\hline$E_{20}$ & $U \oplus E_{8}^{\oplus 2}$ & 18 & 2 & $U$ & $E_{20}$ \\
\hline$Q_{2,0}$ & $U \oplus A_{6} \oplus E_{8}$ & 16 & 4 & $U \oplus C_{8}^{6}$ & $Z_{17}$ \\
\hline$E_{25}$ & $U \oplus E_{7} \oplus E_{8}$ & 17 & 3 & $U \oplus A_{1}$ & $Z_{19}$ \\
\hline$Q_{18}$ & $U \oplus E_{6} \oplus E_{8}$ & 16 & 4 & $U \oplus A_{2}$ & $E_{30}$ \\
\hline
\end{tabular}

Table 4: Picard lattices for lattice mirror symmetric pairs

Proof. For a lattice $L$, denote by $A_{L}$ the discriminant group of $L$, and $q_{L}$ the quadratic form of $L$. We see in Proposition 3.1 that

$$
A_{\operatorname{Pic}(\Delta)} \simeq A_{U \oplus \operatorname{Pic}\left(\Delta^{*}\right)}, \text { and } q_{\operatorname{Pic}(\Delta)}=-q_{U \oplus \operatorname{Pic}\left(\Delta^{*}\right)}
$$

for each case. Thus by Proposition 1.6.1 in [8], there is an isometry

$$
\operatorname{Pic}(\Delta) \simeq\left(U \oplus \operatorname{Pic}\left(\Delta^{*}\right)\right)_{\Lambda_{K 3}}^{\perp}
$$

So $\mathcal{F}_{\Delta}$ and $\mathcal{F}_{\Delta^{*}}$ are lattice mirror symmetric. We shall determine $\operatorname{Pic}(\Delta)$.

1. $Q_{12}$ and $E_{18} \quad$ By Corollary 2.1

$$
\operatorname{Pic}(\Delta) \simeq\left(U \oplus U \oplus A_{2}\right)_{\Lambda_{K 3}}^{\perp} \simeq U \oplus\left(A_{2}\right)_{E_{8}}^{\perp} \oplus E_{8} \simeq U \oplus E_{6} \oplus E_{8} .
$$

2. $Z_{1,0}$ and $E_{19} \quad$ By Corollary 2.1

$$
\operatorname{Pic}(\Delta) \simeq\left(U \oplus U \oplus A_{1}\right)_{\Lambda_{K 3}}^{\perp} \simeq U \oplus\left(A_{1}\right)_{E_{8}}^{\perp} \oplus E_{8} \simeq U \oplus E_{7} \oplus E_{8} .
$$

3. $E_{20}$ and $E_{20}$ Since $\Lambda_{K 3} \simeq U^{\oplus 3} \oplus E_{8}^{\oplus 2}$

$$
\operatorname{Pic}(\Delta) \simeq(U \oplus U)_{\Lambda_{K 3}}^{\perp} \simeq U \oplus E_{8}^{\oplus 2}
$$

4. $Q_{2,0}$ and $Z_{17} \quad$ By Corollary 2.1

$$
\operatorname{Pic}(\Delta) \simeq\left(U \oplus U \oplus C_{8}^{6}\right)_{\Lambda_{K 3}}^{\perp} \simeq U \oplus\left(C_{8}^{6}\right)_{E_{8}}^{\perp} \oplus E_{8} \simeq U \oplus A_{6} \oplus E_{8} .
$$


5. $E_{25}$ and $Z_{19}$ By Corollary 2.1

$$
\operatorname{Pic}(\Delta) \simeq\left(U \oplus U \oplus A_{1}\right)_{\Lambda_{K 3}}^{\perp} \simeq U \oplus\left(A_{1}\right)_{E_{8}}^{\perp} \oplus E_{8} \simeq U \oplus E_{7} \oplus E_{8} .
$$

6. $Q_{18}$ and $E_{30}$ By Corollary 2.1

$$
\operatorname{Pic}(\Delta) \simeq\left(U \oplus U \oplus A_{2}\right)_{\Lambda_{K 3}}^{\perp} \simeq U \oplus\left(A_{2}\right)_{E_{8}}^{\perp} \oplus E_{8} \simeq U \oplus E_{6} \oplus E_{8} .
$$

Thus the assertions are verified.

\section{Application}

Denote by $\Delta_{B}$ the reflexive polytope obtained in [7] for a singularity of type $B$. As is seen in Table 3, there are isometric Picard lattices $\operatorname{Pic}\left(\Delta_{Q_{12}}\right) \simeq \operatorname{Pic}\left(\Delta_{Q_{18}}\right)$, and $\operatorname{Pic}\left(\Delta_{Z_{1,0}}\right) \simeq \operatorname{Pic}\left(\Delta_{E_{25}}\right)$. Families $\mathcal{F}_{\Delta_{B}}$ and $\mathcal{F}_{\Delta_{D}}$ are said to be essentially the same if general members in these families are birationally equivalent. Not only the Picard lattices are isometric, but also we have

Proposition 4.1 The families $\mathcal{F}_{\Delta_{Q_{12}}}\left(\right.$ resp. $\left.\mathcal{F}_{\Delta_{Z_{1}, 0}}\right)$ and $\mathcal{F}_{\Delta_{Q_{18}}}\left(\right.$ resp. $\left.\mathcal{F}_{\Delta_{E_{25}}}\right)$ are essentially the same.

ProOF. It is directly shown that the polytopes $\Delta_{Q_{12}}\left(\operatorname{resp} . \Delta_{Z_{1,0}}\right)$ and $\Delta_{Q_{18}}$ (resp. $\Delta_{E_{25}}$ ) are isometric. Indeed, define an invertible matrix in $G L_{3}(\mathbb{Z})$ as

$$
M_{1}=\left(\begin{array}{ccc}
-1 & 0 & 1 \\
1 & 1 & -2 \\
2 & -3 & 2
\end{array}\right)\left(\text { resp. } M_{2}=\left(\begin{array}{ccc}
-1 & 2 & 1 \\
1 & -3 & -1 \\
2 & 0 & -1
\end{array}\right)\right)
$$

and one obtains an isometry

$$
m M_{1}=m^{\prime}\left(\text { resp. } m M_{2}=m^{\prime}\right)
$$

that sends $m \in \Delta_{Q_{18}}$ to $m^{\prime} \in \Delta_{Q_{12}}$ (resp. $m \in \Delta_{E_{25}}$ to $m^{\prime} \in \Delta_{Z_{1,0}}$ ). Therefore, there exists an explicit projective transformation that maps each Laurent polynomial in $\left|-K_{\mathbb{P}\left(\Delta_{Q_{12}}\right)}\right|$ (resp. $\left|-K_{\mathbb{P}\left(\Delta_{Z_{1,0}}\right)}\right|$ ) to a Laurent polynomial in $\left|-K_{\mathbb{P}\left(\Delta_{Q_{18}}\right)}\right|$ (resp. $\left.\left|-K_{\mathbb{P}\left(\Delta_{E_{25}}\right)}\right|\right)$. This mapping also birationally sends a general member in $\mathcal{F}_{\Delta_{Q_{12}}}$ (resp. $\mathcal{F}_{\Delta_{Z_{1,0}}}$ ) to a general member in $\mathcal{F}_{\Delta_{Q_{18}}}$ (resp. $\left.\mathcal{F}_{\Delta_{E_{25}}}\right)$. Thus the statement is proved.

We conclude our study to remark that general members in compactifications of non-equivalent singularities can be transformed via a reflexive polytope.

\section{References}

[1] Arnol'd, V. I., Critical points of smooth functions and their normal forms, Russian Math. Surveys 30, 1-75 (1975).

[2] Barth, W.P. and Hulek, K. and Peters, C.A.M. and Van de Ven, A., Compact Complex Surfaces, Second ed, Springer (2004).

[3] Dolgachev, I., Mirror symmetry for lattice polarized K3 surfaces, J. Math. Sci. 81, 2599-2630 (1996). 
[4] Ebeling, W. and Ploog, D., A geometric construction of Coxeter-Dynkin diagrams of bimodal singularities, Manuscripta Math. 140, 195-212 (2013).

[5] Iano-Fletcher, A. R., Working with weighted complete intersections, in Explicit Birational Geometry of 3-folds, Alessio Corti and Miles Reid (eds.) London Mathematical Society Lecture Note Series 281, 101-173 (2000).

[6] Kobayashi, M., Duality of weights, mirror symmetry and Arnold's strange duality, Tokyo J. Math., 31, 225-251 (2008).

[7] Mase, M.and Ueda, K, A note on bimodal singularities and mirror symmetry, Manuscripta Math.(online 31 August 2014) 146, 153-177 (2015).

[8] Nikulin, V.V., Integral symmetric bilinear forms and some of their applications, Math. USSR-Izv. 14, 103-167 (1980).

[9] Nishiyama, K., The Jacobian fibrations on some $K 3$ surfaces and their Mordell-Weil groups, Japan J. Math. 22, 293-347 (1996).

[10] Oda, T., Torus Embeddings and Applications, Springer, Tata Institute of Fundamental Research Lectures 57 (1978).

[11] Reid, M., Canonical 3-folds, in Journeés de géométrie algébrique d'Angers, edited by A. Beauville, Sijthoff and Noordhoff, Alphen, 273-310 (1980).

[12] Yonemura, T., Hypersurface simple $K 3$ singularities, Tôhoku Math. J. 42, 351-380 (1990).

e-mail: mtmase@arion.ocn.ne.jp

MAKIKO MASE

Department of Mathematics and Information Sciences, Tokyo Metropolitan University 192-0397 1-1 Minami Osawa, Hachioji-shi, Tokyo, Japan.

Osaka City University Advanced Mathematical Institute

558-8585 3-3-138 Sugimoto-cho, Sumiyoshi-ku, Osaka, Japan. 